Psychology of Language and Communication 2020, Vol. 24, No. 1

\title{
Sciendo
}

DOI: $10.2478 /$ plc-2020-0003

\author{
GAIID LE MANER-IDRISSI ${ }^{1}$, SANDRINE LE SOURN BISSAOUI ${ }^{1}$, \\ VIRGINIE DARDIER ${ }^{1}$, MAXIME CODET ${ }^{2}$, NATHALIE BOTTE-BONNETON ${ }^{1}$, \\ FANNY DELAHAYE ${ }^{1}$, VIRGINIE LAVAL ${ }^{3}$, MARC AGUERT ${ }^{4}$, \\ GÉRALDINE TAN-BESCOND ${ }^{2}$, BENOIT GODEY ${ }^{2}$ \\ ${ }^{1}$ Univ Rennes-Rennes 2- LP3C, Rennes, France \\ ${ }^{2}$ Centre Hospitalier Universitaire, Pontchaillou-Rennes, France \\ ${ }^{3}$ Université de Poitiers-CerCA, Poitiers, France \\ ${ }^{4}$ Université de Caen Basse Normandie, LPCN, Caen, France
}

\section{EMOTIONAL SPEECH COMPREHENSION IN DEAF CHILDREN WITH COCHLEAR IMPLANT}

\begin{abstract}
We examined the understanding of emotional speech by deaf children with cochlear implant (CI). Thirty deaf children with CI and 60 typically developing controls (matched on chronological age or hearing age) performed a computerized task featuring emotional prosody, either embedded in a discrepant context or without any context at all. Across the task conditions, the deaf participants with CI scored lower on the prosody-bases responses than their peers matched on chronological age or hearing age. Additionally, we analyzed the effect of age on determining correct prosody-based responses and we found that hearing age was a predictor of the accuracy of prosody-based responses. We discuss these findings with respect to delay in prosody and intermodal processing. Future research should aim to specify the nature of the cognitive processes that would be required to process prosody.
\end{abstract}

Keywords: Emotional speech, Prosody, Deaf children, Implant cochlear, Pragmatic abilities

\footnotetext{
Address for correspondence: Sandrine Le Sourn Bissaoui, LP3C - Laboratoire de Psychologie, Cognition, Comportement et Communication, University Rennes-Rennes 2, France. E-mail: sandrine.lesourn-bissaoui@univ-rennes2.fr
} 
The Cochlear Implant (CI) is a type of auditory prosthesis that requires surgery to be fitted. An array of electrodes is implanted in the cochlea in order to stimulate the auditory nerve with electrical impulses. CI enables recipients to detect sounds at $3040 \mathrm{~dB}$ (Bond et al., 2009). By allowing profoundly deaf children to perceive and distinguish sounds, CIs provide richer and more accessible input than the classic hearing aids (Bouchard, Ouellet, \& Cohen, 2009; Tomblin, Spencer, Peng, \& Lu, 2008). In this way, they promote interactions between children and their conversational partners. Today, infants with profound bilateral deafness are implanted as young as 6 months (Dettman, Pinder, Briggs, Dowell, \& Leigh, 2007), as a number of studies have not only shown that there are no additional complications following the operation, but have also highlighted the long-term benefits of early implantation (i.e., before 2 or even 1 year of age) for the development of oral language perception and development (Bonns et al., 2013; Dettman et al., 2007; Dillon, de Jong, \& Pisoni, 2012; Manrique, Cervera-Paz, Huarte, \& Molina, 2004; Rinaldi \& Caselli, 2014; Taitelbaum-Swead et al., 2005; Välimaa, Kunari, Laukkanen, \& Lonka, 2018). Their findings indicate that as early as six months post-implant, CI can increase vocal turn-taking, and promote joint attention, topic initiation, and auditory awareness (Tait, de Raeve, \& Nikolopoulos, 2007). At 33 months post-implant, deaf children with CI have markedly different speech act profiles from those that are habitually observed among deaf children (Nicholas, 1994), and the profiles of young cochlear implant users resemble those of younger, normally hearing children. Lastly, researchers have reported steady and significant growth in children's conversational activity during the first year following implantation, as well as an increase in verbal language use as early as the second semester post-activation (Le Maner-Idrissi et al., 2009).

The rapid changes observed in children's receptive and expressive language following implantation might well be linked to the new opportunities for hearing and taking part in daily conversations afforded by CI. These implants allow deaf children to enjoy some of the natural exposure to verbal language that enables hearing children to acquire language (Robbins, Svirsky, \& Kirk, 1997). However, as suggested recently by Tomaszewski, Krzysztofiak and Moroń (2019), although CI technology can support speech development by improving hearing skills and assisting with acquisition of spoken language as the first language, it cannot fully guarantee that the expected outcomes will be achieved. Currently, the literature reports mixed findings regarding the ability of deaf children with cochlear implants to "catch up" to their hearing peers' level of vocabulary knowledge, particularly with regard to spoken vocabulary (see Convertino, Borgna, Marschark, \& Durkin, 2014; Nicholas \& Geers, 2007; Nott, Cowan, Brown, \& Wigglesworth, 2009). 


\section{Prosody in Emotional Speech and Cochlear Implantation}

Understanding emotional speech is a complex skill that plays a major role in social cognition (Jiam, Caldwell, Deroche, Chatterjee, \& Limb, 2017). Most of the research among cochlear implant users has focused on comprehension, intelligibility, and production of speech, with reduced attention to speech prosody (Hegarty \& Faulkner, 2013; Jiam et al., 2017). However, speech conveys not only linguistic content, but also information-through emotional prosodyabout the speaker's emotional state, whether happy or sad (Marx et al., 2015), and about communicative intentions such as humor or irony (Nakata, Trehub, $\&$ Kanda, 2012). Prosodic distinctions result from the perception of variations in fundamental frequency (F0) or pitch, duration and amplitude of speech (Nakata et al., 2012). Happy utterances, for example, are higher and more variable in pitch, louder and faster than sad utterances (Banse \& Scherer, 1996; Nakata et al., 2012). Pitch assists listeners in recognizing the gender, identity and emotional state of the speaker (Clearly, Pisoni, \& Kirk, 2005; Hegarty \& Faulkner, 2013). Current CI technology does not permit the perception of the fine-grained spectral and temporal detail needed to perceive F0 and pitch (Drennan \& Rubinstein, 2008; Moein, Khoddami, \& Shahbodaghi, 2017; Straatman, Rietveld, Beijen, Mylanus, \& Mens, 2010; Torppa et al., 2014; Zeng, 2004).

From the age of 3 or 4 years, hearing children are able to infer a speaker's emotional state from his/her emotional prosody (see Baltaxe, 1991; Stifter \& Fox, 1987). There has been scant research on the perception of prosody in implanted children, but the perception of prosody has always been reported as limited (Jiam et al., 2017).

Studies on the ability of CI users to identify emotion in speech have shown that they have difficulty recognizing vocal emotions because they have impaired access to the fine structure in speech (Hopyan, Manno, Papsin, \& Gordon, 2015; Kalathottukaren, Purdy, \& Ballard, 2015; See, Driscoll, Gfeller, Kliethermes, \& Oleson, 2013). Luo, Fu and Galvin (2007) played sentences conveying happiness, sadness, anger, anxiety, and emotional neutrality to deaf adult CI users aged 4173 years. CI users identified less than half $(44.9 \%)$ the emotions, which is much lower than the performance of normal hearing adults (89.8\%). In the same way, Agrawal et al. (2013) compared the performances of deaf adults fitted with CIs and hearing adults on the recognition of prosody in sentences (neutral, angry, happy). Performance accuracy scores indicated that the CI users had difficulty recognizing prosody (65\% correct responses), compared with hearing adults (95\% correct responses). With children, Wiefferink, Rieffe, Ketelaar and Frijns (2012) examined two aspects of emotional understanding: Recognition of emotion in facial expressions (happy, sad, angry, fearful) and recognition of emotion in a situational context. Results showed that emotional comprehension was poorer 
in CI users than in hearing children. Similarly, studies have shown that prelingually deaf 7 - to 13 -year-olds with unilateral implants can identify emotion in faces (happy, sad, angry, fearful), but have more difficulty than hearing children in perceiving affective speech prosody (Hopyan-Misakyan, Gordon, Dennis, \& Papsin, 2009). When Chin, Bergeson and Phan (2012) studied emotional moods (declarative, interrogative, happy or sad) in deaf children $(M=8.31$ years) with unilateral implants, they found that hearing children achieved a higher number of correct responses on measures of prosody (Prosodic Utterance Production Test) than CI users. In the same way, Volkova, Trehub, Schellenberg, Papsin and Gordon (2013) studied the ability of 5- to 7-year-old deaf children with bilateral CIs to identify happiness and sadness in speech. Results indicated that their ability was well above random chance level but remained significantly poorer than that of their hearing peers. Nonetheless, results for CI users showed higher achievement than in previous research. This could be attributed to bilateral implantation, which provides the most salient pitch cues and the most functional spectral resolution. The authors, however, attributed these results to the fewer alternative responses available and to the use of child-directed speech.

However, because the vocal expression of emotion always co-occurs with the verbal expression of emotions and affects, researchers have extended the number of cues they consider. Emotional speech comprehension presupposes that the individual grasps the emotional valence of an utterance by relying on various cues produced by the speaker. In order to understand emotional speech, the listener has to process not only the cues produced by the speaker's words, but also the associated contextual cues. In typical development, research has shown that adults rely primarily on emotional prosody to understand emotional speech, unlike preschoolers and schoolchildren up to 9 years (Aguert, Laval, Le Bigot, \& Bernicot, 2010), who rely more on semantic content (Friend \& Bryant, 2000; Morton \& Trehub, 2001; Waxer \& Morton, 2011) or situational context (Aguert et al., 2010). Aguert et al. investigated the understanding of emotional speech independently of lexical content in conditions where emotional prosody was discrepant with the situational context. Using a computerized paradigm based on a judgment task, they showed that there was a developmental transition in the use of cues to infer the speaker's intention, with a shift from situational context as the overriding cue (at ages 5 and 7 years) to emotional prosody (adults and children from 9 years old). This transition appeared to begin at age 9, with 9-yearolds relying on both types of cue (situational context and emotional prosody). Although when prosody was the sole cue, they all managed to use this cue to infer the speaker's intention. Moreover, Aguert et al. (2010) and Le Sourn-Bissaoui, Aguert, Girard, Chevreuil, and Laval (2013) have demonstrated the existence of contextual and negative bias in emotional 
speech comprehension for both typical and atypical trajectories (autism spectrum disorders). Contextual bias is a tendency to rely on the situational context, rather than the emotional prosody, to infer the speaker's intention even when the situational context was discrepant with prosody. Negative bias is a propensity to attend to, learn from, and use negative information far more than positive information.

The above results showed that children with cochlear implants had difficulty perceiving prosody in situations in which the context is not taken into account. In this study, the objective will be to examine the role of situational context and emotional prosody in emotional speech comprehension of deaf children with CIs. Using the computerized paradigm developed by Aguert et al. (2010), this paper aims to examine the understanding of emotional speech in CI population.

\section{Present Study}

The aim of this research was to examine the role of emotional prosody in the comprehension of emotional speech in deaf children fitted with CIs, depending on whether this prosody is alone or embedded in a situational context.

First, we should describe processing prosody across conditions (prosody alone or embedded in a situational context) in the deaf children with CI group and the typical children group. So we postulated that CI users have difficulty understanding emotional speech, maybe because of an emotional prosody processing impairment since CIs decrease the ability to perceive emotion in speech, meaning that $\mathrm{CI}$ users have difficulty perceiving expressive variations in speech. We investigated the ability of deaf children with CIs to infer the speaker's intent from emotional prosody with or without a situational context, and independently of lexical content. More specifically, we compared the ways in which deaf children with CIs and their hearing peers use emotional prosody to infer the speaker's intention, either when the emotional prosody is the only available cue or when the emotional prosody and the situational context convey discrepant information about the speaker's intention. The question we raised was: Do deaf children with CI have lower performance than their controls? Do the CI users have the same strategy to process emotional prosody according to context or prosody valence than their controls?

Additionally, we analyzed the effect of age on performance. According to Aguert et al. (2010), relying on the situational context is typical of young children, whereas adults rely uppermost on the prosody. We, therefore, investigated the relationship between age and processing prosody. The more precise question we raised was: Do hearing age or chronological age predict children's performance? We postulated the dominant effect of hearing age, in other words, the time since implantation, on the ability to infer the speaker's intent from emotional prosody. 
To test these research questions, we elaborated an experimental design with an original experimental paradigm applied by Aguert et al. (2010), which allows for investigating the evaluation of emotional prosody in situational context.

\section{Method}

\section{Participants}

A total of 90 middle-class children took part in this research. They were divided into three groups: 30 participants fitted with CIs (CI group), 30 participants matched on chronological age with the CI group (CA controls) and 30 participants matched on hearing age (age at implantation) with the CI group (HA controls). The second and third groups therefore constituted a comparison sample of 60 typically developing controls with no learning difficulties and no developmental disorders.

The CI group comprised 30 children (18 boys and 12 girls) with profound prelingual deafness. These children had all been implanted with MED-EL Opus II devices. Their mean age at implantation was 2 years 3 months $(S D=9 \text { months, range }=10 \text { months to } 4 \text { years })^{1}$. At the date of the study, their mean chronological age was 9 years 6 months $(S D=2$ years 4 months, range $=5$ years 3 months to 13 years), and their mean hearing age was 7 years 3 months $(S D=2$ years, range $=3$ years 6 months to 10 years). No disorders were associated with the deafness, and there was a good level of homogeneity within the sample in terms of age at implantation, type of implant used (MED-EL Opus II) and therapy (auditory-verbal). None of the deaf children had oral communication (spoken French), and they used LSF (French Sign Language) before implantation. The aetiologies of hearing loss were genetic (42\%), unknown (38\%), auditory neuropathy (two children), meningitis (one child) and ototoxicity (one child). All of them had been implanted at Pontchaillou University Hospital in Rennes, France.

The second group (CA controls) were 30 typically developing controls matched with the CI group on sex and chronological age $\left(M_{\mathrm{CI}}=9\right.$ years 6 months, $S D=2$ years 4 months, range $=5$ years 3 months to 13 years; $M_{\text {CAcontrols }}=9$ years 5 months, $S D=2$ years 4 months, range $=5$ years 1 month to 13 years 2 months; $t(58)=0.12, p=.90)$.

The third group (HA controls) were 30 typically developing controls matched with the CI group on sex and hearing age $\left(M_{\mathrm{CI}}=7\right.$ years 3 months, $S D=2$ years, range $=3$ years 6 months to 10 years; $M_{\text {HAcontrols }}=7$ years 3 months, $S D=1$ years 9 months, range $=4$ years 6 months to 10 years 2 months; $t(58)=-0.14, p=.88)$.

\footnotetext{
${ }^{1}$ All the children with profound prelingual deafness were enrolled in a treatment protocol at Rennes University Hospital, France, encompassing their medical, paramedical and psychological follow-up. It was at this hospital that all the children had been fitted with their CIs.
} 
All the participants came from French-speaking homes and their parents had normal hearing. All children (CI, HA and CA groups) attended mainstream preschool, primary or secondary schools in France. None of the children were reported to have cognitive or learning difficulties. Receptive (oral comprehension) language was assessed using the ELO test (Oral Language Evaluation, Khomsi, 1999). A qualified speech-language pathologist administered the test orally. The ELO test comprised 21 items for children under 10 years and 32 items for children over 10 years. Each correct response scored 1 point. To compare scores independently of chronological age, we calculated a quotient of oral comprehension, dividing each child's score by the total possible score, resulting in a quotient between 0 and 1. CI participants were found to have a significantly lower verbal quotient than their hearing peers matched on CA $\left(M_{\text {CIparticipants }}=0.87, \mathrm{SD}=0.17 ; M_{\text {CAparticipants }}=0.96\right.$, $S D=0.08 ; t(58)=-.263, p<.05)$ but not than their hearing peers matched on HA $\left(M_{\text {Clparticipants }}=0.94, S D=0.09 ; M_{\text {HAparticipants }}=0.94, S D=0.09 ; t(58)=-1.93\right.$, $p=.85)$.

All participants were given an oral outline of the procedure. Written informed consent was obtained from their parents. The study was approved by the ethics committee and conducted in accordance with the Declaration of Helsinki (1975).

\section{Material and Procedure}

Computerized paradigm. To examine the role of emotional prosody in the comprehension of emotional speech in children, we used PILOU's computerized paradigm (Aguert et al., 2010). The strong point of this paradigm is that it simulates conversations between two characters that are attractive for children, Pilou the rabbit and Edouard the duck. In this paradigm, twelve meaningless utterances conveying emotional prosody were played to all the participants. The emotional prosody is either embedded in a non-discrepant context or stands alone. Aguert et al. constructed the utterances by randomly combining common syllables in French. Each utterance contains five syllables. The situational context is presented both in a picture form and by a prerecorded off-screen voice.

The emotional valence of the prosody was pre-tested acoustically and psychologically. Although there have been a great many studies on the subject, it is still quite difficult to define discrete basic emotions, such as sadness or joy, in terms of a particular pattern of the three acoustic parameters of prosody, namely fundamental frequency (F0), intensity, and duration (Juslin \& Laukka, 2003; Spackman, Brown, \& Otto, 2009). This is why Aguert et al. (2010) chose to adopt a dimensional approach (Laukka, Juslin, \& Bresin, 2005; Russell, Bachorowski, \& Fernandez-Dols, 2003) to contrast the prosody of otherwise meaningless utterances. They first checked the prosodic contrast of the utterances on the valence (negative vs. positive) 
dimension. A group of 17 naive adults were asked to rate the prosody of the meaningless utterances on a 5-point scale ranging from 0 (very negative) to 5 (very positive). The mean score of the utterances conveying positive prosody was $4.63(S D=0.25)$, compared with $1.26(S D=0.22)$ for the utterances conveying negative prosody, $t(16)=37.85, p<.001$. The authors then checked the prosodic contrast of the 12 utterances on the activation (high vs. low energy) dimension by conducting an acoustic analysis with PRAAT software (Boersma \& Weenink, 2004). Particular attention was paid to F0, the main parameter reflecting the activation of an emotion in prosody (Bänziger \& Scherer, 2005; Liberman \& Michaels, 1962; Quam \& Swingley, 2012; Scherer, 1986). A low, monotonous F0 pattern is characteristic of negative emotions in their depressed form (e.g., sadness, cold anger, disgust) whereas a high, wide-range F0 pattern is characteristic of positive emotions in their excited form (e.g., happiness, excitement, elation) (Scherer, 1986). The F0 analysis of the utterances showed that the positive prosody did indeed contrast strongly with the negative prosody on this parameter. The former had a high mean F0 $(M=262 \mathrm{~Hz}, S D=23.5 \mathrm{~Hz})$, indicating high activation, and the latter a lower mean F0 $(M=135 \mathrm{~Hz}, S D=15.1 \mathrm{~Hz}), t(10)=11.15, p<.001$, indicating low activation. Moreover, positive prosody was characterized by a wide F0 range $(M=39.5 \mathrm{~Hz}, S D=9.3 \mathrm{~Hz})$, and negative prosody by a lower, more monotonous F0 range $(M=13.0 \mathrm{~Hz}, S D=5.9 \mathrm{~Hz}), t(10)=5.89, p<.001$.

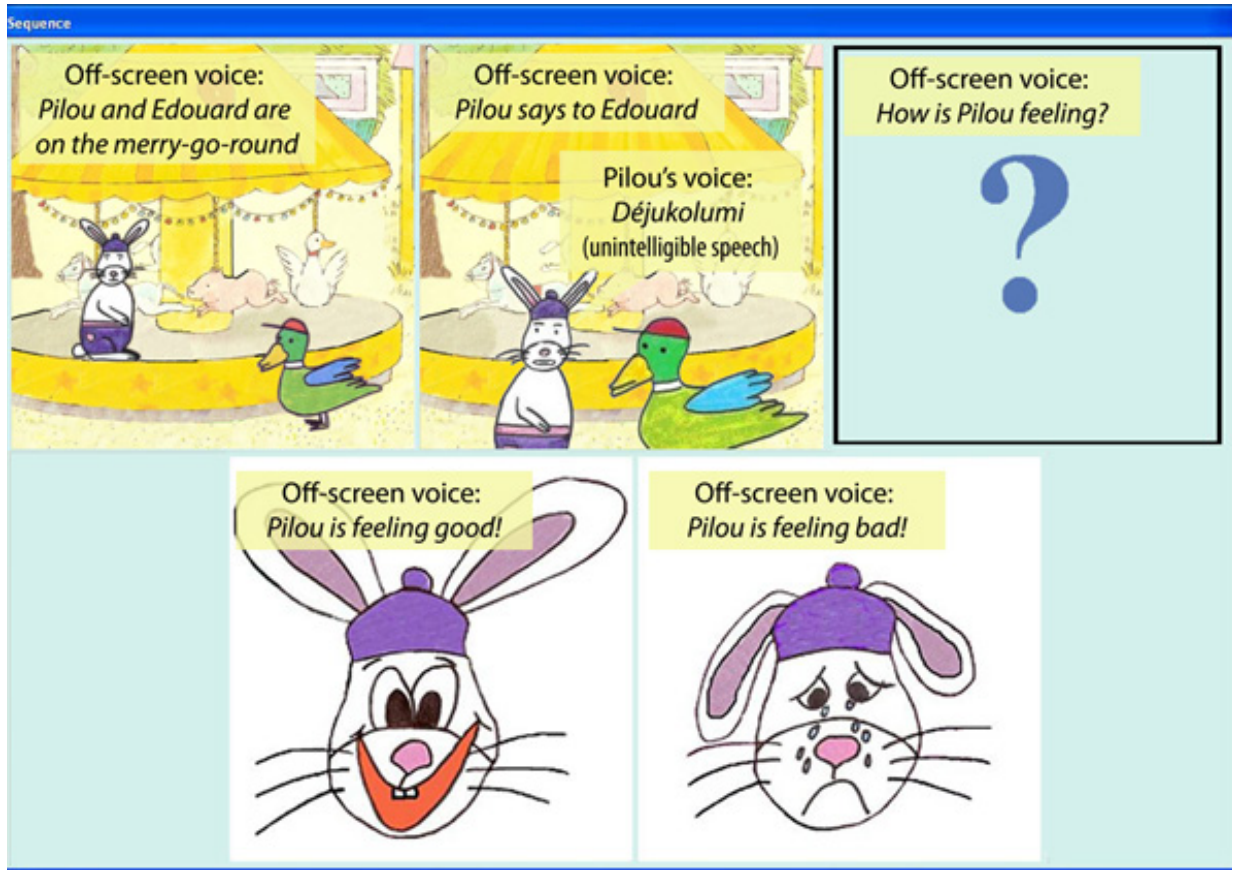

Figure 1. Screen capture of a story just before the participant's response (yellow boxes added to show the audio side of the story). 
The emotional valence of the situational contexts was then pre-tested with a different sample of 28 adults, who had to judge it on a 5-point scale ranging from 1 (totally negative) to 5 (totally positive). The six negative situational contexts were judged to be very negative $(M=1.52, S D=0.40)$, and the positive situational contexts very positive $(M=4.49, S D=0.34)$, $t(27)=26.11, p<.001$.

For the condition involving prosody embedded in context, we used 12 stories about the adventures of Pilou the rabbit and Edouard the duck in everyday situations as constructed by Aguert et al. (2010). Each story, set in a given situational context, leads to an interaction between the two characters in which Pilou the rabbit expresses a meaningless utterance to Edouard the duck. The 12 situational contexts and 12 meaningless utterances conveying the emotional prosody which, together, made up the 12 stories, were either positive or negative. The 12 experimental stories varied according to the situational context (positive vs. negative) and the emotional valence of the prosody (positive vs. negative). By combining these two factors, we constructed four types of situation: a negative situational context with positive emotional prosody $(\mathrm{C}-\mathrm{P}+)$, a positive situational context with negative emotional prosody $(\mathrm{C}+\mathrm{P}-)$, a negative situational context with negative emotional prosody (C-P-), and a positive situational context with positive emotional prosody $(\mathrm{C}+\mathrm{P}+)$. These four types of situation corresponded to two types of story: discrepant situation stories (C-P+ and $\mathrm{C}+\mathrm{P}-)$ and non-discrepant situation stories $(\mathrm{C}+\mathrm{P}+$ and $\mathrm{C}-\mathrm{P}-)$. In this study, we analysed the responses given by participants for the discrepant situation stories, and used the non-discrepant stories as fillers and controls. For the condition involving prosody without emotional context, the 12 meaningless utterances were presented without any situational context.

For all conditions, after the expressive meaningless utterance, the child had to answer the question, "So what do you think, how is Pilou feeling?" on two possible responses: Pilou is feeling good or Pilou is feeling bad. The child touched the screen to choose his/her answer (see Figure 1).

Each participant was tested individually in a quiet room, where the experimenter settled him or her in front of the computer. The deaf children with CIs were tested in a clinical setting while the CA and HA participants were tested in a school setting. In the first of two sessions, participants were asked to judge the prosody embedded in a context. In the second session, the prosody was presented on its own. One week separated the two sessions.

Data analyses. In this study, the prosody (positive vs. negative) was either embedded in a discrepant context or presented on its own. We, therefore, obtained four ( $2 \times 2)$ experimental conditions: positive prosody alone, positive prosody embedded in a discrepant context, negative prosody alone, and negative prosody embedded in a discrepant context. In each condition, we analysed the prosody-based responses given by the participants. There were 
two possible responses: Pilou is feeling good or Pilou is feeling bad. A prosody-based response was a response compatible with the prosody (i.e., Pilou is feeling good when the prosody was positive, and Pilou is feeling bad when the prosody was negative).

In the principal research question, we should describe processing prosody in the deaf children with CI group and the typical children group across conditions. Thus, response (prosody-based vs. non-prosody-based) was the dependent variable. Group (CI vs. CA controls vs. HA controls), condition (no context vs. discrepant context) and prosody valence (positive vs. negative) were the fixed factors. The first operational hypothesis postulated a main effect of group, that the CI group will score lower on the task across all the contextual and prosody valence conditions. Previous researches investigating emotional speech comprehension using the same computerized paradigm have shown contextual and negative bias in typical children (Aguert et al., 2010) and in atypical children (autism spectrum disorders: Le Sourn-Bissaoui et al., 2013). To examine the understanding of emotional speech in the CI population, we also studied whether the CI population has the same strategy to process emotional prosody as their hearing controls. Therefore, the second operational hypothesis postulated that the proportion of prosody-based responses would be higher in the no-context condition than in the discrepant context condition (contextual bias), while the third operational hypothesis postulated that the proportion of prosody-based responses would also be higher when the prosody was negative than when it was positive (negative bias).

In the additional research question, we analyzed the effect of age on performances and postulated that hearing age would predict the proportion of prosody-based responses, such that older children, in terms of hearing age, would choose more prosody-based responses in all conditions (no context vs. discrepant) and for all prosody valence.

In this study, we used the non-discrepant stories as fillers and controls. In the non-discrepant condition, a correct response (i.e., context matching with the emotional prosody) was awarded 1 point and otherwise, no point were attributed. Each participant judged six items $(3 \mathrm{P}+\mathrm{C}+$ and $3 \mathrm{P}-\mathrm{C}-)$, so the maximum score was 6 . Figure 2 presents the number of participants in each group who gave correct responses in the prosody embedded in context: no discrepant situation condition. Kruskal-Wallis test was conducted to examine the differences between the three groups. Where the emotional prosody was associated with a non-discrepant context, when all six items $(\mathrm{P}+\mathrm{C}+$ and $\mathrm{P}-\mathrm{C}-)$ were considered, we found significant differences between the three groups, $\mathrm{K}(2)=12.14, p=0.002$. Post hoc tests indicated that the CA group differed from both the HA group $(p=.001)$ and the CI group $(p=0.005)$. However, there was no significant difference between the CI group and the HA group $(p=.0728)$. Thus, the CA group exhibited better scores than both, the CI and HA groups. 


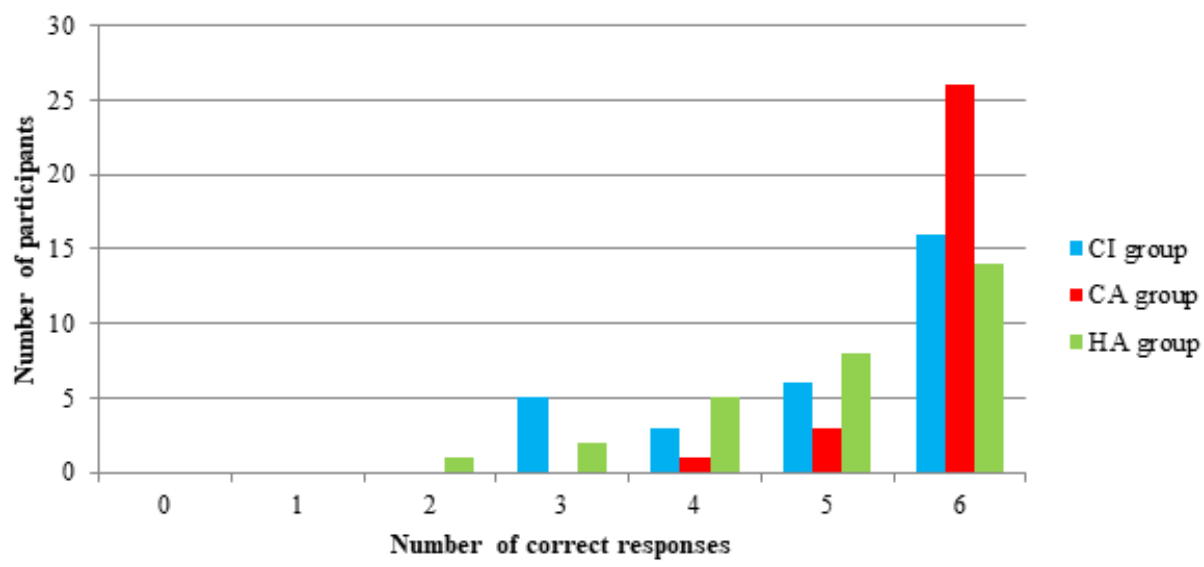

Figure 2. Number of participants in each group (Cochlear Implant group, Chronological Age Group and Hearing Group) who gave correct responses in the prosody embedded in context: no discrepant situation condition.

\section{Results}

The SPSS software programs were used to conduct all analyses on the data.

\section{Comparison of CI Participants and Controls}

A generalized estimating equation (GEE) approach was used to investigate the predictive value of the independent variables. This method, an extension of traditional linear repeated-measures models, serves to analyze non-normally distributed data (e.g., binary categorical). Response (prosody-based vs. non-prosody-based) was the dependent variable. The fixed factors were: Group (CI vs. CA controls vs. HA controls), condition (no context vs. discrepant context) and prosody valence (positive vs. negative).

Table 1. Means (Standard Errors) of Overall Proportion of Prosody-Based Responses Depending on the Group, Condition and Prosody Valence

\begin{tabular}{lcccc}
\hline & \multicolumn{2}{c}{ No context } & \multicolumn{2}{c}{ Discrepant context } \\
\hline & Positive & Negative & Positive & Negative \\
CI participants & $0.68(.02)$ & $0.66(.02)$ & $0.22(.03)$ & $0.28(.03)$ \\
CA participants & $0.95(.02)$ & $0.93(.02)$ & $0.62(.04)$ & $0.74(.04)$ \\
HA participants & $0.93(.03)$ & $0.97(.03)$ & $0.77(.04)$ & $0.53(.03)$ \\
\hline
\end{tabular}

Table 1 (means and standards errors) and Figure 3 (with the 95\% confidence interval) presents statistics descriptive of the overall proportion of prosodybased responses according to group, conditions, and valence. The GEE analysis revealed a significant group effect, Wald $\chi^{2}(2)=65.91, p<.001$. The proportion of prosody-based responses was lower for CI participants (45\%) than for CA controls $(80.5 \%, p<.001)$ and HA controls $(79.25 \%, p<.001)$. 
Moreover, differences between CA controls and HA controls were not statistically significant (Wald $\chi^{2}(1)=.05, p=1$ ). As predicted, the CI group exhibited lower scores in the prosody task than both groups of match controls. The analysis also revealed a significant effect of condition (Wald $\chi^{2}(1)=$ $77.48, p<.001)$. This effect indicated that the proportion of prosody-based responses was higher in the no-context condition $(85.16 \%)$ than in the discrepant context condition $(51.33 \%)$. This finding was consistent with the contextual bias hypothesis. Furthermore, results indicated a significant effect of prosody valence (Wald $\chi^{2}(1)=5.52, p<.01$ ). The proportion of prosody-based responses was higher when the prosody was negative $(70.60 \%)$ than when it was positive $(65.80 \%)$. This finding was consistent with the negative bias hypothesis.

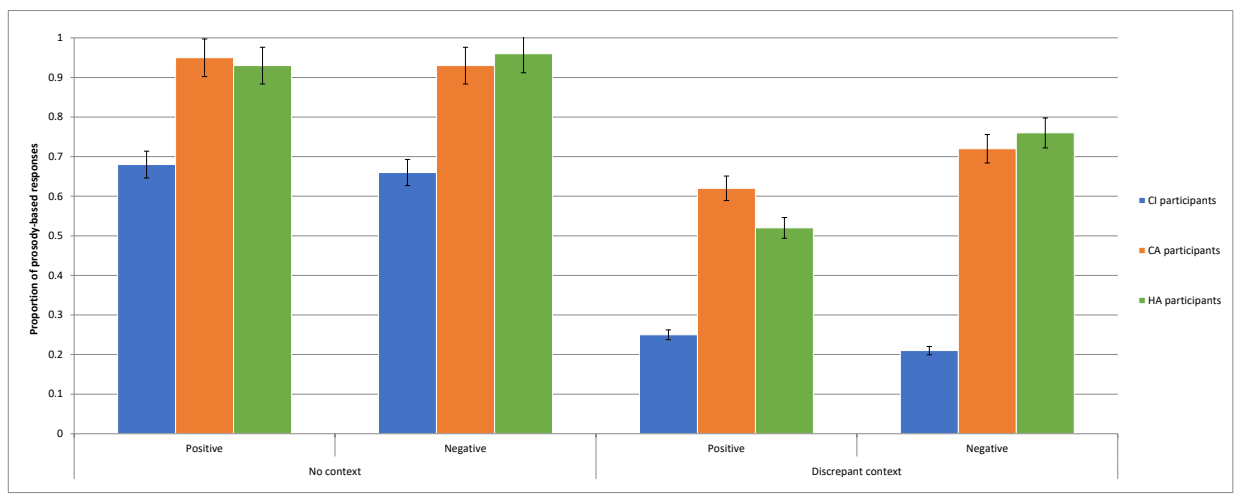

Figure 3. Overall proportion of prosody-based responses depending on the group (Cochlear Implant group, Chronological Age Group and Hearing Group), the condition and the prosody valence. Errors bars show the $95 \%$ confidence interval.

None of the Group x Condition (Wald $\chi^{2}(2)=.82, p=.66$ ), Group $\mathrm{x}$ Prosody valence (Wald $\chi^{2}(2)=4.09, p=.12$ ) or Group $\mathrm{x}$ Condition $\mathrm{x}$ Prosody valence interactions reached statistical significance (Wald $\chi^{2}(1)=.95, p=.62$ ). This suggests that the groups did not differ in their patterns of responses.

\section{Did the Participants Perform Above Chance Level?}

Because the participants had only two possible choices, there was a 50:50 chance of choosing a prosody-based response. We, therefore, conducted an additional qualitative analysis to check that the participants did not perform the task at random. We sorted participants according to their responses to the six stories in the discrepant context (see Table 2). Those participants who gave four, five or six prosody-based responses out of six were designated as prosody-based participants. Those who gave zero, one or 
two prosody-based responses (out of 6) were designated as context-based participants. Those who gave three prosody-based responses out of six were designated as random participants.

Table 2. Number of "Prosody-Based," "Random" and "Context-Based" Participants According to Group

\begin{tabular}{lccc}
\hline & Prosody-based & Random & Context-based \\
\hline CI group & 2 & 5 & 23 \\
CA controls group & 19 & 2 & 9 \\
HA controls group & 16 & 9 & 5 \\
\hline
\end{tabular}

This qualitative analysis showed that none of the groups performed the task randomly. Only a few participants (5/30 for CI group; $2 / 30$ for CA control group; $9 / 30$ for HA control group) randomly chose how the speaker was feeling, and the majority (25/30 for CI group; 28/30 for CA control group; $21 / 30$ for HA control group) consistently relied on a cue, be it prosody or context. A $\chi^{2}$ test showed that the distribution of the CI participants differed significantly from that of the CA controls, $\chi^{2}(2)=20, p<.001$ and HA controls, $\chi^{2}(2)=24, p<.001$. CI participants consistently relied on context, whereas the hearing participants (CA and HA controls) relied more on prosody.

\section{Impact of Age on Performance}

According to Aguert et al. (2010), relying on the situational context is typical of young children, whereas adults rely uppermost on the prosody. Our main concern was the relation between age (hearing age or chronological age) and the degree of prosody-based responses in various conditions (no context vs. discrepant context). Hearing age is calculated from the date of implantation for deaf children and from birth for their controls. Chronological age is calculated from birth for all children. Our operational hypothesis postulated that hearing age would predict the proportion of prosody-based responses such that older children, in terms of hearing age, would choose more prosodybased responses in all conditions (no context vs. discrepant) and for all prosody valence (positive vs. negative).

This strategy would allow us to predict the contribution of these two factors (hearing age and chronological age) to a change in the prosody-based response scores, firstly in a no-context condition and secondly in a discrepant context condition.

Table 3 presents the results of the stepwise regression analyses we conducted on the prosody-based response scores with the following predictors: hearing age and chronological age. The regression coefficients shown are those at the final step. The first two analyses assessed the contribution of hearing age and chronological age to variability in prosody-based responses scores in the no-context condition. The results obtained showed that the variability in children's performance was only predicted by hearing age 
$\left(R^{2}=0.17, F(1,89)=17,89 p<.001\right)$. Chronological age was removed in the final model predicting performances in the condition involving prosody without context. The second analysis assessed the same two factors against variability in children's performance in the discrepant context condition. These results indicated that only hearing age made the primary contribution to the prediction of change in children's performance in the discrepant context condition $\left(R^{2}=0.07, F(1,89)=6,16, p<.05\right)$. Chronological age was removed in the final model predicting performances in the condition involving prosody embedded in context. Our finding was consistent with our main hypothesis, according to which, hearing age is a predictor of the ability to infer the speaker's intent from emotional prosody.

Table 3. Summary of Stepwise Regression Analyses Predicting Prosody-Based Responses from Hearing Age and Chronological Age According to Conditions (No-Context vs. Discrepant Context) (N=90)

\begin{tabular}{lcccc}
\hline Variable/step & $\mathbf{B}$ & $\mathbf{S E}_{\mathbf{B}}$ & Beta & $\mathbf{R}^{\mathbf{2}}$ \\
\hline $\begin{array}{l}\text { No context condition } \\
\text { Final step }\end{array}$ & .034 & .008 & .41 & $.17^{* * * *}$ \\
$\quad$ Hearing age & & & & \\
Discrepant context condition & & & & $.07 *$ \\
Final step & .02 & .008 & .26 &. \\
$\quad$ Hearing age & & & & \\
\hline
\end{tabular}

Note: $* \mathrm{p}<.05, * * * \mathrm{p}<.001$

\section{Discussion}

The purpose of this study was to examine the understanding of emotional speech by deaf children fitted with CIs. To this end, we used a computerized paradigm (Aguert et al., 2010) to simulate communication situations. We first described processing prosody across the conditions (prosody alone or embedded in a situational context) in the deaf children with CI group and the typical children group, and, additionally, studied the effect of age on performances.

Our first concern was to compare the ways in which deaf children with CIs and their hearing peers use emotional prosody to infer the speaker's intention, either when the emotional prosody is the only available cue or when the emotional prosody and the situational context convey discrepant information about the speaker's intention. The first question we raised was: Do deaf children with CI have lower performance than their controls? We hypothesized first that the prosody-based responses would be lower for CI participants than for their CA or HA controls across all of the contextual and prosody valence conditions. The second question we raised was: Do deaf children with CI population have the same strategy to process emotional prosody? Therefore, we postulated that 
the proportion of prosody-based responses would be higher in the no-context condition than in the discrepant context condition (contextual bias), and also when the prosody was negative than when it was positive (negative bias). As expected, we found a lower performance for CI participants than for their CA or HA controls in all conditions and for all prosody valence. Moreover, the CI participants and their hearing controls exhibited the same pattern of processing, giving fewer prosody-based responses when the prosody was embedded in a discrepant context than when it was on its own, and giving more prosody-based responses when the prosody was negative than when it was positive. With the same computerized paradigm (Aguert et al., 2010), in recent studies (Laval, Dardier, Laval, \& Monetta, 2016; Aguert et al., 2010; Le Sourn-Bissaoui et al., 2013) negative bias and contextual bias seems to be a consistent result observed across different age groups (adults vs. children) and in both, typical and atypical development. Our study, using the same procedure and the same computerized paradigm, seems to provide the first evidence for the existence of this contextual and negative bias in a CI population. For contextual bias, our results shed light on typical development. Aguert et al. (2010) showed that a developmental switch occurs at around 9 years, with prosody beginning to override the situational context as the primary cue to understanding the speaker's intention. The 9-year-olds in this study relied both on emotional prosody and on situational context, in contrast to adults, who relied solely on emotional prosody. Our results showed that the analysis of communication situations, and specifically, the simultaneous consideration of contextual and prosodic cues, was a difficult exercise for the implanted deaf children in our experimental group. This is probably because these situations require a more sustained level of inferential processing. The responses of the children fitted with CIs were based more on context. They gave priority to the indicator that was the most prominent, most available, and most familiar to them (i.e., the context), whereas the hearing children's responses were based more on prosody. Our study showed that, compared with the situational context, emotional prosody conveyed less salient information for the deaf children with CI. For negative bias, our results can be explained by the asymmetrical way in which humans use positive and negative information to make sense of their world (Vaish, Grossman, \& Woodward, 2008). More specifically, adults display a negativity bias, that is, a propensity to attend to, learn from, and use negative information far more than positive information. For example, the negativity bias has repeatedly been observed in adults' judgment- and decision-making (for a review, see Peeters \& Czapinski, 1990). When making judgments, people consistently lend greater weight to the negative aspects of an event or stimulus than to the positive aspects (Kahneman \& Tversky, 1984). In development, most studies 
have confirmed this asymmetry in young children, particularly in areas of emotional development such as social referencing (Mumme, Fernald, $\&$ Herrera, 1996) and emotional contagion (Vaish et al., 2008).

Additionally, we explored the contribution of age to emotional prosody process. We hypothesized that hearing age, or the time since implantation, should predict the proportion of prosody-based responses with older children (in terms of hearing age), choosing prosody-based responses more frequently in all conditions (no context vs. discrepant context) and for all prosody valence (positive vs. negative). Our finding was consistent with our principal hypothesis, according to which, hearing age is a predictor of the ability to infer the speaker's intent from emotional prosody. Thus, as expected, the first factor that could explain the weaker performance of the deaf participants with $\mathrm{CI}$ is the time since implantation. Children who had experienced a longer period of sound deprivation had been given fewer opportunities to hear emotional prosody, and thence fewer opportunities than their hearing controls to detect this prosody. Speech prosody is characterized by variations in duration, intensity, and pitch (Cutler, Dahan, \& van Donselaar, 1997; Langus, Marchetto, Bion, Hoffmann, \& Nespor, 2012; Lehiste, 1970). According to Hopyan, Gordon and Papsin (2011), speech perception also involves the processing of information conveyed by emotional prosody. This prosody is characterized by various parameters such as the tonal pitch, scope and direction, and rhythmicity (Ladd, 1996). For Hopyan et al., optimum oral communication involves being able to hear both speech and emotional prosody. According to Volkova et al. (2013), changes in pitch or intonation help people to identify emotions. These parameters are most likely to pose difficulties for implanted children. Moreover, beyond the detection of prosodic signals, hearing children learn, from a very early age, how to decode the uses of prosody in conversational contexts. They are exposed to emotional prosody long before they utter their first words. As soon as a child is born, adults can convey information about their emotional state to him or her in everyday interactions by modulating their prosody (Papoušek, 1992). Recent research has highlighted the difficulties that deaf children with CIs have in identifying emotions in others' speech (Hopyan-Misakyan et al., 2009; Luo et al., 2007; Most \& Aviner, 2009). Finally, our results support the notion that the difficulties experienced by implanted children in understanding emotional speech stems from their difficulties in drawing appropriate inferences, especially in multiple-cue environments. Given that implanted deaf children have less experience in using emotional prosody, it is possible that their weaker results are due to a developmental lag.

Further research is necessary to investigate the weaker performance of implanted deaf children. In this respect, other than hearing age, the first factor that could explain the poorer performance of implanted deaf children is a reduced level of basic receptive prosodic ability (inferring a speaker's 
intention from emotional prosody). In our research, the proportion of prosodybased responses was lower for deaf participants with cochlear implants than for controls, both in the discrepant situation and when prosody was the sole cue. Thus, as Hopyan-Misakyan et al. (2009) or Most (2016) suggest, despite the fact that, from a technical point of view, CIs are becoming more sophisticated in detecting sound, their ability to process emotional prosody is still limited. Accordingly, implanted deaf children cannot optimally hear variations in intonation, volume, and tempo. Emotional prosody takes a long time to become salient during typical development (Quam \& Swingley, 2012). In implanted deaf children's development, it could take longer still, owing to their weaker basic receptive ability. Future research will have to specify the nature of this difficulty in inferring the speaker's intention from emotional prosody, in terms of intermodal difficulties and/or technical prosody perception. In addition, as suggested by Most (2016), future research should examine the effects of various kinds of sensory aid (hearing aid, cochlear implant or bimodal stimulation) on an individual's perceptions of prosody based on suprasegmental and paralinguistic features. The second factor that could explain the poorer performance of implanted deaf children would be one or more neurocognitive processes, such as executive functioning. According to Kronenberger, Colson, Henning and Pisoni (2014), executive functioning may influence the development of speech-language skills in deaf children after cochlear implantation, in ways that differ from their hearing peers. In our computerized paradigm, processing emotional prosody in a context, especially in a discrepant context, would require cognitive control. In typical development, Aguert et al. (2010) showed a contextual bias. Thus, in the discrepant context, choosing the response based on emotional prosody requires inhibiting the response based on context. the inhibition skills that enable the non-relevant and more salient information to be ignored (the discrepant context) and allow the participant to react just on the emotional prosody. Recent research has highlighted that preschoolers with CIs showed significantly poorer performance with respect to inhibition-concentration and working memory when compared with their hearing peers (Beer et al., 2014). These differences in measured performances in terms of executive functioning remained when data was controlled for language. Thus, future research should aim to specify the nature of the cognitive processes that would be required to process prosody.

The present study highlights the need to consider several factors involved in social interactions (context, prosody, emotions). Future research will need to investigate the links between them. Achim, Guitton, Jackson, Boutin and Monetta (2013) recently highlighted the complexity of the factors involved in the attribution of mental states in situations of everyday interaction. Their model incorporated various sources of information, some in the immediate environment (immediate perception of the speaker, the utterance's occurrence, 
and the situational and linguistic context), others stored in memory (about the person, people in general, and the immediate context). In a conversational situation, the recipient of a message must be able to simultaneously process all the information that is received. This processing requires complex intermodal integration (Barkhuysen, Krahmer, \& Swerts, 2010). More research with implanted deaf children is needed to identify their intermodal processing strategies. The results of our research also highlighted the need to conduct longitudinal studies to take different possible developmental paths into account. A longitudinal study would help to establish the age at which the transition to adult processing takes place in both, typical and atypical development. Additional studies are, therefore, needed if we want to gain a better understanding of emotional speech and track comparative developmental trajectories in typical and CI populations. These longitudinal studies are particularly relevant today, as the age of children at the time of implantation continues to decrease. Moreover, it would be interesting to continue studying the developmental trajectories of deaf children fitted with CIs to assess their changing skills in emotional prosody processing in relation to their hearing age and their chronological age, as the level of verbal understanding does not seem to be the main explanation for the differences observed between implanted children and their controls. This longitudinal perspective should yield new information on the development of communication in deaf children fitted with CIs.

This study has several implications in terms of assessment and intervention. The computerized paradigm (Aguert et al., 2010) we used could contribute to efforts to understand why the CI population has difficulties understanding emotional speech, as it enables researchers to create situational contexts. This computerized paradigm could prove helpful in assessing difficulties in the CI population by making it possible to distinguish between a basic receptive prosodic ability (inferring a speaker's intention from emotional prosody) and a more sophisticated ability (inferring a speaker's intention from emotional prosody in a situational context). Our study highlights the need to study language comprehension with an ecological task that is appropriate and attractive for children. This finding is also an important point to consider when designing speech therapy programs. Clinicians could program specific interventions based on receptive prosodic ability or inferential ability in order to develop the comprehension of indirect language, such as utterances based on prosodic cues (e.g., irony, sarcasm) in an ecological situation (Happé, 1994). According to Hopyan-Misakyan et al. (2009), given the initial lack of stimulation in terms of emotional prosody, it is possible that the brain development of children with CI differs from that of children with hearing. It is also possible that the reception of affective prosodic stimuli could gradually improve through CI use, promoting cortical reorganization and offering new possibilities for information processing. These phenomena will 
be the subject of future research. We should also underline the heterogeneity of the results for the implanted children, with some of them performing better than others. It would certainly be worthwhile for future studies to focus on the variability we observed here, as identifying the characteristics of the best performers would provide useful pointers for improving the care of all implanted deaf children.

In conclusion, from a clinical perspective, this future research would contribute to a better understanding of the subtle difficulties observed in the development of communication in implanted deaf children as well as with their rehabilitation in a speech therapy context. From a theoretical perspective, this research may also shed light on our understanding of the cognitive processes involved in understanding language in context. The rapid changes observed in children's receptive and expressive language following implantation may well be linked to the new opportunities for hearing and taking part in daily conversations afforded by CIs. These implants allow deaf children to enjoy some of the natural exposure to verbal language that enables hearing children to acquire language (Robbins, Svirsky, \& Kirk, 1997). However, as implanted deaf children are also deprived of language before they are fitted with their CIs, they may not receive the interactive information they need to understand expressions of emotion (Rieffe \& Meerum Terwogt, 2000). In fact, a link has been found between delays in the language development of no-implant deaf children with hearing parents and difficulties in emotional comprehension (Meerum Terwogt \& Rieffe, 2004; Moeller, Tomblin, Yoshinaga-Itano, Connor, \& Jerger, 2007). In fact, the role of emotions is a fundamental factor in the social development of deaf children with cochlear implants. Their emotional skills are considered as being in continuous development through social interactions with their environment. From this point of view, interacting with people by understanding their emotional prosody is an essential social ability in humans. The ability to analyze complex socio-emotional indices enables a better regulation of interactions with others and a better social integration of deaf children with cochlear implants. One practical implication of this study is that therapeutic interventions for deaf children should expand their focus on the suprasegmental aspects of speech perception and production, especially intonation. Moreover, the research suggests that music would be a valuable tool for the rehabilitation of implanted children. Recent studies have found that engagement in music was linked with better performance across a range of measures (Torppa et al., 2014; Wang, Trehub, Volkova, \& van Lieshout, 2013). The findings of the present study also underscore the need for further development of processing strategies for $\mathrm{CI}$ users in order to achieve an accurate representation of pitch, which is essential for the correct perception of prosody. 


\section{Acknowledgments}

We thank Camille Bonnet and Valérie Fremont for data collection.

\section{References}

Achim. A. M., Guitton, M., Jackson, P., Boutin, A., \& Monetta, L. (2013). On what ground do we mentalize? Characteristics of current tasks and sources of information that contribute to mentalizing judgments. Psychological Assessments, 25, 117-126. doi:10.1037/a0029137

Agrawal, D., Thorne, J. D., Viola, F. C., Timm, L., Debenar, S., Büchner, A., ... Wittfoth, M. (2013). Electrophysiological responses to emotional prosody perception in cochlear implant users. NeuroImage: Clinical, 2, 229-238. doi:10.1016/j.nicl.2013.01.001

Aguert, M., Laval, V., Le Bigot, L., \& Bernicot, J. (2010). Understanding expressive speech acts: The role of prosody and situational context in French-speaking 5- to 9-year-olds. Journal of Speech, Language, and Hearing Research, 53(6), 1629-1641.

Baltaxe, C. A. M. (1991). Vocal communication of affect and its perception in three- to four-year-old children. Perceptual and Motor Skills, 72, $1187-1202$.

Banse, R., \& Scherer, K. R. (1996). Acoustic profiles in vocal emotion expression. Journal of Personality and Social Psychology, 70(3), 614-636. doi:10.1037/0022-3514.70.3.614

Bänziger, T., \& Scherer, K. R. (2005).The role of intonation in emotional expressions. Speech Communication, 46, 252-267.

Barkhuysen, P., Krahmer, E., \& Swerts, M. (2010). Crossmodal and incremental perception of audiovisual cues to emotional speech. Language and Speech, $53,3-30$.

Beer, J., Kronenberger, W. G., Castellanos, I., Colson, B. G., Henning, S. C., \& Pisoni, D. B. (2014). Executive functioning skills in preschool-age children with cochlear implants. Journal of Speech, Language, and Hearing Research, 57(4), 1521-1534.

Boersma, P., \& Weenink, D. (2004). PRAAT: Doing phonetics by computer. Retrieved from www.praat.org

Bond, M., Mealing, S., Anderson, R., Elston, J., Weiner, G., Taylor, R. S., ... Stein, K. (2009). The effectiveness and cost-effectiveness of cochlear implants for severe to profound deafness in children and adults: A systematic review and economic model. Health Technology Assessment, 13(44), 1-330. doi:10.3310/hta13440 
Bonns, T., de Raeve, L., Langereis, M., Peeraer, L., Wouters, J., \& van Wieringen, A. (2013). Expressive vocabulary, morphology, syntax and narrative skills in profoundly deaf children after cochlear implantation. Research in Developmental Disabilities, 34(6), 2008-2022.

Bouchard, M. -È., Ouellet, C., \& Cohen, H. (2009). Speech development in prelingually deaf children with cochlear implants. Language and Linguistics Compass, 3, 1-18.

Chin, S. B., Bergeson, T. R., \& Phan, J. (2012). Speech intelligibility and prosody production in children with cochlear implants. Journal of Communication Disorders, 45, 355-366. doi:10.1016/j.jcomdis.2012.05.003

Clearly, M., Pisoni, D. B., \& Kirk, K. I. (2005). Influence of voice similarity on talker discrimination in children with normal hearing and children with cochlear implants. Journal of Speech, Language and Hearing Research, 48, 204-223.

Convertino, C., Borgna, G., Marschark, M., \& Durkin, A. (2014). Word and world knowledge among deaf learners with and without cochlear implants. Journal of Deaf Studies and Deaf Education, 19(4), 471-483.

Cutler, A., Dahan, D., \& van Donselaar, W. (1997). Prosody in the comprehension of spoken language: A literature review. Language and Speech, 40(2), 141-201.

Dettman, S. J., Pinder, D., Briggs, R. J. S., Dowell, R. C., \& Leigh, J. R. (2007). Communication development in children who receive the cochlear implant younger than 12 months: Risks versus benefits. Ear and Hearing, 28(2), 11-18.

Dillon, C. M., de Jong., K., \& Pisoni, D. B. (2012). Phonological awareness, reading skills, and vocabulary knowledge in children who use cochlear implants. Journal of Deaf Studies Deaf Education, 17(2), 205-226.

Drennan, W. R., \& Rubinstein, J. T. (2008). Music perception in cochlear implant users and its relationship with psychophysical capabilities. Journal of Rehabilitation Research and Development, 45, 779-789.

Friend, M., \& Bryant, J. B. (2000). A developmental lexical bias in the interpretation of discrepant messages. Merrill-Palmer Quarterly, 46(2), 342-369.

Happé, F. (1994). An advanced test of theory of mind: Understanding of story characters' thoughts and feelings by able autistic, mentally handicapped, and normal children and adults. Journal of Autism and Developmental Disorders, 24(2), 129-154.

Hegarty, L., \& Faulkner, L. (2013). The perception of stress and intonation in children with a cochlear implant and a hearing aid. Cochlear Implants International, 14, 35-39. doi:10.1179/1467010013Z.000000000132

Hopyan, T. M., Gordon, K. A., \& Papsin, B. C. (2011). Identifying emotions in music through electrical hearing in deaf children using cochlear implants. Cochlear Implants International, 12, 21-26. 
Hopyan, T. M., Manno, F. A., Papsin, B. C., \& Gordon, K. A. (2015). Sad and happy emotion discrimination in music by children with cochlear implants. Child Neurolpsychology: A Journal on Normal and Abnormal Development in childhood and Adolescence, 6(3), 1-15. doi:10.1080/0929 7049.2014.992400

Hopyan-Misakyan, T. M., Gordon, K. A., Dennis, M., \& Papsin, B. C. (2009). Recognition of affective speech prosody and facial affect in deaf children with unilateral right cochlear implants. Child Neuropsychology, 15, 136-146. doi:10.1080/0929704080240368

Jiam, N. T., Caldwell, M., Deroche, M. L., Chatterjee, M., \& Limb, C. J. (2017). Voice emotion perception and production in cochlear implant users. Hearing Research, 352, 30-39.

Juslin, P. N., \& Laukka, P. (2003). Communication of emotions in vocal expression and music performance: Different channels, same code? Psychological Bulletin, 129, 770-814.

Kahneman, D., \& Tversky, A. (1984). Choices, values, and frames. American Psychologist, 39, 341-350.

Kalathottukaren, R. T., Purdy, S. C., \& Ballard, E. (2015). Prosody perception and musical pitch discrimination in adults using cochlear implants. International Journal of Audiology, 54, 444-452. doi:10.3109/14992027.2014.997314

Khomsi, A. (1999). Lecture de mots et compréhension-révisée: LMC-R, épreuve d'évaluation de la compétence en lecture. E.C.P.A. Paris

Kronenberger, W. G., Colson, B. G., Henning, S. C., \& Pisoni, D. B. (2014). Executive functioning and speech-language skills following long-term use of cochlear implants. Journal of Deaf Studies and Deaf Education, 19(4), $456-470$.

Ladd, D. R. (1996). International Phonology. Cambridge: Cambridge University Press.

Langus, A., Marchetto, E., Bion, R., Hoffmann, A., \& Nespor, M. (2012). Journal of Memory and Language, 66(1), 285-306.

Laval, V., Dardier, V., Laval, C., \& Monetta, L. (2016). La compréhension de l'intention d'autrui est-elle sous l'influence d'un biais négatif? Bulletin de Psychologie, 2, 117-126.

Laukka, P., Juslin, P. N., \& Bresin, R. (2005). A dimensional approach to vocal expression of emotion. Cognition \& Emotion, 19, 633-653.

Lieberman, P., \& Michaels, S. B. (1962). Some Aspects of Fundamental Frequency and Envelope Amplitude as Related to the Emotional Content of Speech. Journal of the Acoustical Society of America, 34, 922-927.

Lehiste, I. (1970). Suprasegmentals. Cambridge: MIT Press.

Le Maner-Idrissi, G., Rouxel, G., Pajon, C., Dardier, V., Baligand, Z., Tan-Bescond, G., \& Godey, B. (2009). Cochlear implant and lexical diversity development in deaf children: Intra- and interindividual differences. Current Psychology Letters, 25(2), 1-13. 
Le Sourn-Bissaoui, S., Aguert, M., Girard, P., Chevreuil, C., \& Laval, V. (2013). Emotional speech comprehension in children and adolescents with autism spectrum disorders. Journal of Communication Disorders, 46(4), 309-320

Luo, X., Fu, Q. J., \& Galvin, J. (2007). Vocal emotion recognition by normal-hearing listeners and cochlear implant users. Trends in Amplification, $11,301-315$.

Manrique, M., Cervera-Paz, F. J., Huarte, A., \& Molina, M. (2004). Prospective long-term auditory results of cochlear implantation in prelinguistically deafened children: The importance of early implantation. Acta Oto Laryngologica. Supplement, 552, 55-63.

Marx, M., James, C., Foxton, J., Capber, A., Fraysse, B., Barone, P., \& Deguine, O. (2015). Speech prosody perception in cochlear implant users with and without residual hearing. Ear and Hearing, 36, 239-248. doi:0196/0202/2015/362-0239/0

Meerum Terwogt, M., \& Rieffe, C. (2004). Behavioral problems in deaf children: Theory of mind delay or communication failure? European Journal of Developmental Psychology, 1, 231-240. doi:10.1080/ 17405620444000139

Moein, N., Khoddami, S. M., \& Shahbodaghi, M. R. (2017). A comparison of speech intonation production and perception abilities of Farsi speaking cochlear implanted and normal hearing children. International Journal of Pediatric Otorhinolaryngology, 101, 1-6. doi:10.1016/j.ijporl.2017.07.018

Moeller, M. P., Tomblin, J. B., Yoshinaga-Itano, C., Connor, C. M., \& Jerger, S. (2007). Current state of knowledge: Language and literacy of children with hearing impairment. Ear and Hearing, 28, 740-753. doi:10.1097/AUD.060113318157f07f

Morton, J. B., \& Trehub, S. E. (2001). Children's understanding of emotion in speech. Child Development, 72(3), 834-843.

Most, T. (2016). Perception of the prosodic characteristics of spoken language by individuals with hearing loss. In M. Marschark, P. E. Spencer (Eds.), The Oxford Handbook of Deaf Studies in Language (pp. 79-93). Oxford: Oxford University Press.

Most, T., \& Aviner, C. (2009). Auditory, visual, and auditory-visual perception of emotions by individuals with cochlear implants, hearing aids, and normal hearing. Journal of Deaf studies and Deaf Education, 14, 449-464. doi:10.1093/deafed/enp007

Mumme, D. L., Fernald, A., \& Herrera, C. (1996). Infants' responses to facial and vocal emotional signals in a social referencing paradigm. Child Development, 67, 3219-3237.

Nakata, T., Trehub, S. E., \& Kanda, Y. (2012). Effect of cochlear implants on children's perception and production of speech prosody. The Journal of the Acoustical Society of. America, 131, 1307-1314. doi:10.1121/1.3672697 
Nicholas, J. G. (1994). Sensory aid use and the development of communicative function. The Volta Review, 96, 181-198.

Nicholas, J. G., \& Geers, A. E. (2007). Will they catch up? The role of age at cochlear implantation in the spoken language development of children with severe to profound hearing loss. Journal of Speech, Language, and Hearing Research, 50(4), 1048-1062.

Nott, P., Cowan, R., Brown, P. M., \& Wigglesworth, G. (2009). Early language development in children with profound hearing loss fitted with a device at a young age: Part I-The time period taken to acquire first words and first word combinations. Ear and Hearing, 30(5), 526-540.

Papoušek, M. (1992). Early ontogeny of vocal communication in parent-infant interactions. In: Papoušek, H., Jürgens, U., Papoušek, M. (Eds.) Nonverbal vocal communication: Comparative and developmental approaches (pp. 230-261). New York: Cambridge University Press.

Peeters, G., \& Czapinski, J. (1990). Positive-negative asymmetry in evaluations: The distinction between affective and informational negativity effects. European Review of Social Psychology, 1, 33-60.

Quam, C., \& Swingley, D. (2012). Development in children's interpretation of pitch cues to emotions. Child Development, 83(1), 236-250. doi:10.1111/j.1467-8624.2011.01700

Rieffe, C., \& Meerum Terwogt, M. (2000). Deaf children's understanding of emotions: Desires take preference. Journal of Child Psychology and Psychiatry, 41, 601 pp. 230-261 608. doi:10.1017/S0021963099005843

Rinaldi, P., \& Caselli, M. C. (2014). Language development in a bimodal bilingual child with cochlear implant: A longitudinal study. Bilingualism: Language and Cognition, 17(4), 798-809.

Robbins, A. M., Svirsky, M., \& Kirk, K. I. (1997). Children with implants can speak, but can they communicate? American Academy of Otolaryngology, 17(3), 155-160.

Russell, J. A., Bachorowski, J.-A., \& Fernandez-Dols, J.-M. (2003). Facial and vocal expressions of emotion. Annual Review of Psychology, 54, 329.

Scherer, K. R. (1986). Vocal affect expression: a review and a model for future research. Psychological Bulletin, 99, 143-165.

See, R. L., Driscoll, V. D., Gfeller, K., Kliethermes, S., \& Oleson, J. (2013). Speech intonation and melodic contour recognition in children with cochlear implants and with normal hearing. Otology and Neurotology, 34(3), 490-498. doi:10.1097/MAO.0b013e318287c985

Spackman, M. P., Brown, B. L., \& Otto, S. (2009). Do emotions have distinct vocal profiles? A study of idiographic patterns of expression. Cognition and Emotion, 23, 1565-1588.

Stifter, C. A., \& Fox, N. A. (1987). Preschool children's ability to identify and label emotions. Journal of Nonverbal Behavior, 11, 43-54. 
Straatman, L. V., Rietveld, A. C., Beijen, J., Mylanus, E. A. M., \& Mens, L. H. M. (2010). Advantage of bimodal fitting in prosody perception for children using a cochlear implant and a hearing aid. The Journal of the Acoustical Society of America, 128, 1884-1895.

Tait, M., de Raeve, L., \& Nikolopoulos, T. P. (2007). Deaf children with cochlear implants before the age of 1 year: Comparison of preverbal communication with normally hearing children. International Journal of Pediatric Otorhinolaryngology, 71, 1605-1611.

Taitelbaum-Swead, R., Kishon-Rabin, L., Kaplan-Neeman, R., Muchnik, C., Kronenberg, J., \& Hildesheimer, M. (2005). Speech perception of children using Nucleus, Clarion or Med-El cochlear implants. International Journal of Pediatric Otorhinolaryngology, 69(12), 1675-1683.

Tomaszewski P., Krzysztofiak, P., \& Moroń, E. (2019). From sign language to spoken language? A new discourse of language development in deaf children. Psychology of Language and Communication, 23(1), 48-84.

Tomblin, J. B., Spencer, S. C., Peng, L. J., \& Lu, N. (2008). Long-term trajectories of the development of speech sound production in pediatric cochlear implant recipients. Journal of Speech Language and Hearing Research, 51(5), 1353-1368.

Torppa, R., Faulkner, A., Huotilainen, M., Järvikivi, J., Lipsanen, J., Laasonen, M., \& Vainio, M. (2014). The perception of prosody and associated auditory cues in early-implanted children: The role of auditory working memory and musical activities. International Journal of Audiology, 53, 182-191. doi:10. 3109/14992027.2013.872302

Vaish, A., Grossmann, T., \& Woodward. A. (2008). Not all emotions are created equal: The negativity bias in social-emotional development. Psychological Bulletin, 134(3), 383-403.

Välimaa, T., Kunnari, S., Laukkanen, N. P., \& Lonka, E. (2018). Early vocabulary development in children with bilateral cochlear implants. International Journal of Language \& Communication Disorders, 53(1), 3-15.

Volkova, A., Trehub, S. E., Schellenberg, E. G., Papsin, B. C., \& Gordon, K. A. (2013). Children with bilateral cochlear implants identify emotion in speech and music. Cochlear Implants International, 14(2), 8091. doi:101179/1754762812Y00E000004

Wang, D. Y., Trehub, S. E., Volkova, A., \& van Lieshout, P. (2013). Child implant users' imitation of happy- and sad-sounding speech. Frontiers in Psychology, 4, 1-8. doi:10.3389/fpsyg.2013.00351

Waxer, M., \& Morton, J. B. (2011). Children's judgments of emotion from conflicting cues in speech: Why 6-year-olds are so inflexible. Child Development, 82(5), 1648-1660. doi:10.1111/j.1467-8624.2011.01624.x 
Wiefferink, C. H., Rieffe, C., Ketelaar, L., \& Frijns, J. H. M. (2012). Predicting social functioning in children with a cochlear implant and in normal-hearing children: The role of emotion regulation. International Journal of Pediatric Otorhinolaryngology, 76, 883-889. doi:10.1016/j. ijporl.2012.02 065

Zeng, F. G. (2004). Trends in cochlear implants. Trends in Amplification, 8, 1-34. 